QUARTERLY OF APPLIED MATHEMATICS

VOLUME LXVII, NUMBER 4

DECEMBER 2009, PAGES 725-734

S 0033-569X(09)01159-5

Article electronically published on May 14, 2009

\title{
A NONLOCAL QUENCHING PROBLEM ARISING IN A MICRO-ELECTRO MECHANICAL SYSTEM
}

\author{
BY \\ JONG-SHENQ GUO (Department of Mathematics, National Taiwan Normal University, 88, S-4, \\ Ting Chou Road, Taipei 11677, Taiwan; and Taida Institute of Mathematical Sciences, National \\ Taiwan University, 1, S-4, Roosevelt Road, Taipei 10617 Taiwan), \\ BEI HU (Department of Mathematics, University of Notre Dame, Notre Dame, Indiana 46556), \\ AND \\ CHI-JEN WANG (Department of Mathematics, National Taiwan Normal University, 88, S-4, Ting \\ Chou Road, Taipei 11677, Taiwan)
}

Abstract. In this paper, we study a nonlocal parabolic problem arising in the study of a micro-electro mechanical system. The nonlocal nonlinearity involved is related to an integral over the spatial domain. We first give the structure of stationary solutions. Then we derive the convergence of a global (in time) solution to the maximal solution as the time tends to infinity. Finally, we provide some quenching criteria.

1. Introduction. In this paper, we study the following nonlocal parabolic problem:

$$
\begin{cases}u_{t}=u_{x x}-\lambda g(t ; u) u^{-2}(x, t), & -1<x<1, t>0 \\ u( \pm 1, t)=1, & t>0, \\ u(x, 0)=u_{0}(x), & -1 \leq x \leq 1\end{cases}
$$

where $\lambda>0, u_{0}(x)$ is a smooth function such that $0<u_{0}(x) \leq 1$ for all $x \in[-1,1]$ and $u_{0}( \pm 1)=1$, and

$$
g(t ; u):=\left(1+\chi \int_{-1}^{1} u^{-1}(x, t) d x\right)^{-2}
$$

for a given positive constant $\chi$. Without loss of generality, by a suitable re-scaling, we shall assume that $\chi=1$ throughout this paper. Furthermore, we suppose that the initial datum $u_{0}$ is symmetric with respect to $x=0$.

This problem arises in the modeling of an elastic membrane suspended above a rigid plate with a fixed voltage source and a fixed capacitor, by assuming the ratio of the

Received July 23, 2008

2000 Mathematics Subject Classification. Primary 35K60, 35Q72, 34B18.

Key words and phrases. Quenching, nonlocal parabolic problem, micro-electro mechanical system.

The first author was partially supported by the National Science Council of the Republic of China under the grant NSC 96-2119-M-003-001.

(C)2009 Brown University 
inertial terms and the damping terms is zero and the deflection $u$ is only of one dimension. The parameter $\lambda$ is defined by

$$
\lambda=\frac{V^{2} L^{2} \epsilon_{0}}{2 l^{2} \tau}
$$

where $V$ is the applied voltage, $\tau$ is the tension in the membrane, $L$ is a characteristic length of the domain, $l$ is a characteristic width of the gap between the membrane and the fixed electrode, and $\epsilon_{0}$ is the primitivity of free space. We refer to 15] for a derivation of the equation. See also 6, 14 for related models in MEMS.

We say that a solution $u$ of (1.1) quenches, if there exists a finite $T$ such that

$$
\liminf _{t \uparrow T}\left\{\min _{-1 \leq x \leq 1} u(x, t)\right\}=0 .
$$

In this case, the nonlocal term becomes singular and the solution no longer exists as a classical solution after $T$, the so-called quenching time. In electrical engineering, it is the so-called pull-in instability phenomenon. Therefore, it is very important to study whether the solution of (1.1) quenches in finite time.

The study of quenching has drawn a lot of attention following the work of Kawarada [12] in 1975. The main questions in these works are quenching criteria, quenching locations, quenching rate, and spatial profile at quenching time. For the local problem with general negative power, i.e., for the equation

$$
u_{t}=u_{x x}-\sigma u^{-\beta}, \quad \sigma, \beta>0,
$$

we refer the reader to [13] for the quenching criteria, 8] for the quenching locations, [8, 4, 9] for the quenching rates, and [5] for the quenching profile.

One of the main features of equation (1.1) is the nonlocal nature of the functional $g(t, u)$. One of the main tools for studying blow-up and quenching properties is the comparison principle. Notice that if the term $u^{-1}(x, t)$ in (1.2) is replaced by any positive power of $u(x, t)$, then the comparison principle would still hold true. In this case, the monotonicity in time of the solution can be derived for certain initial data. But for the system (1.1), unfortunately, the comparison principle is not valid.

For the system (1.1), the question of when the solution quenches in finite time, the quenching criterion, is closely related to the structure of stationary solutions. In section 2 , we shall prove that a constant $\lambda^{*}>0$ exists such that there are no positive stationary solutions of (1.1) if $\lambda>\lambda^{*}$; there is exactly one positive stationary solution of (1.1) if $\lambda=\lambda^{*}$; and there are two positive stationary solutions of (1.1) if $\lambda<\lambda^{*}$. Moreover, the minimum of the stationary solution is decreasing as $\lambda$ increases for the maximal solution branch, and is increasing as $\lambda$ increases for the minimal solution branch for $\lambda \in\left(0, \lambda^{*}\right]$.

In section 3 , we shall give some global (in time) solutions and derive the convergence of these global solutions to the maximal stationary solutions as $t \rightarrow \infty$. Finally, we provide some quenching criteria for $\lambda$ sufficiently large in section 4 . In particular, we show that there is a cusp at the quenching point $x=0$ under certain conditions on the initial data. 
2. Stationary solutions. In this section, we study positive solutions of the following boundary value problem:

$$
\begin{aligned}
& U_{x x}=\lambda\left(1+\int_{-1}^{1} U^{-1}(x) d x\right)^{-2} U^{-2},-1<x<1, \\
& U( \pm 1)=1
\end{aligned}
$$

for a given positive constant $\lambda$.

Note that a solution of the nonlocal problem (2.1)-(2.2) must be a solution of the following local boundary value problem:

$$
w_{x x}=\sigma w^{-2},-1<x<1, \quad w( \pm 1)=1,
$$

where, obviously,

$$
\sigma=\lambda\left(1+\int_{-1}^{1} U^{-1}(x) d x\right)^{-2}, \quad \text { if } U=w .
$$

Notice that some properties and the structure of solutions of (2.3) have been studied in detail by Levine (cf. [13]). For example, the problem (2.3) has only classical solutions. These solutions must be strictly convex and symmetric with respect to $x=0$. Hence there exists a unique minimum point at $x=0$. Moreover, there is a positive constant $\sigma^{*}$ such that there are exactly two solutions of (2.3) if $0<\sigma<\sigma^{*}$, there is exactly one solution of (2.3) if $\sigma=\sigma^{*}$, and there are no solutions of (2.3) if $\sigma>\sigma^{*}$. We denote by $u_{\lambda}$ a solution of (2.1)-(2.2) for a given $\lambda$, and $w_{\sigma}$ a solution of (2.3) for a given $\sigma$. Then $u_{\lambda}=w_{\sigma}$ with $\lambda=\lambda(\sigma)$. It follows from (2.4) that

$$
\lambda=\lambda(\sigma)=\sigma\left(1+\int_{-1}^{1} w_{\sigma}^{-1}(x) d x\right)^{2}
$$

for some $\sigma \in\left(0, \sigma^{*}\right]$.

We can obtain some information for $\lambda$ by investigating the relation (2.5). Since there are two solutions for each $\sigma<\sigma^{*}$, it is not so obvious as to how one can obtain the exact information for $\lambda$ from (2.5). See also [15] for an analysis of this relation. In this paper, we shall use a different approach which is motivated by the work of Deng [2]. We also refer the reader to 1, 2, 3, 10, 11 for some related works on nonlocal parabolic problems.

Suppose that $U(x)$ is a solution of (2.1)-(2.2). Recall that $U(x)$ has exactly one minimum point at $x=0$. Let

$$
F_{1}(s)=-s^{-1}, \quad G_{1}(\mu)=\int_{\mu}^{1} \frac{1}{\sqrt{F_{1}(s)-F_{1}(\mu)}} d s,
$$

where $\mu=U(0) \in(0,1)$. Then

$$
G_{1}(\mu)=\frac{\sqrt{2 \lambda}}{1+Y}
$$

where $Y=\int_{-1}^{1} U^{-1}(x) d x$. Indeed, multiplying the equation (2.1) by $U_{x}$ and integrating it over $[0, x]$ for any $x \in[0,1]$, we obtain that

$$
\frac{1}{2}\left[U_{x}(x)\right]^{2}=-\lambda(1+Y)^{-2}\left[U(x)^{-1}-\mu^{-1}\right], \quad x \in[0,1] .
$$


Since $U_{x}>0$ in $(0,1]$, it follows from (2.7) that

$$
\frac{U_{x}(x)}{\sqrt{\mu^{-1}-U(x)^{-1}}}=\frac{\sqrt{2 \lambda}}{1+Y}, \quad x \in(0,1] .
$$

Hence (2.6) can be deduced by integrating (2.8) from 0 to 1 and using the boundary condition (2.2).

In order to derive a relation between $\lambda$ and $\mu$ which is independent of $Y$, we let

$$
y=\frac{2 \int_{-1}^{x} U^{-1}(s) d s}{Y}-1, \quad h(y)=1+\ln \{U(x)\}, \quad-1 \leq x, y \leq 1 .
$$

Then $h(y)$ satisfies

$$
h_{y y}=\lambda Y^{2} /[2(1+Y)]^{2} \exp [1-h(y)],-1<y<1,
$$

and $h( \pm 1)=1$. Similarly, $h(y)$ has exactly one minimum $\nu$ at 0 , where $\nu=1+\ln \mu$. Now, let

$$
F_{2}(s)=-\exp \{-s\}, \quad G_{2}(\nu)=\int_{\nu}^{1} \frac{1}{\sqrt{F_{2}(s)-F_{2}(\nu)}} d s
$$

Using the same process as above, we have

$$
G_{2}(\nu)=\sqrt{\frac{\lambda e}{2}} \frac{Y}{1+Y} .
$$

It follows from (2.6) and (2.9) that

$$
Y=\frac{2}{\sqrt{e}} \frac{G_{2}(\nu)}{G_{1}(\mu)} .
$$

Since $\nu=1+\ln \mu$, substituting (2.10) into (2.6), we get

$$
\lambda=\frac{1}{2}\left(G_{1}(\mu)+\frac{2}{\sqrt{e}} G_{2}(\nu)\right)^{2}:=K(\mu) .
$$

Next, by changing variables, we claim that

$$
\begin{aligned}
& G_{1}(\mu)=\sqrt{\mu} \sqrt{1-\mu}+\mu^{3 / 2} \ln \frac{\sqrt{1-\mu}+1}{\sqrt{\mu}}, \\
& G_{2}(\nu)=2 \sqrt{\mu e} \ln \frac{\sqrt{1-\mu}+1}{\sqrt{\mu}}, \quad \nu=1+\ln \mu .
\end{aligned}
$$

Recall that

$$
G_{1}(\mu)=\int_{\mu}^{1} \frac{1}{\sqrt{F_{1}(s)-F_{1}(\mu)}} d s=\int_{\mu}^{1} \frac{\sqrt{\mu s}}{\sqrt{s-\mu}} d s, \mu \in(0,1) .
$$


Set $s=\mu \sec ^{2} \vartheta$. Then we obtain

$$
\begin{aligned}
G_{1}(\mu) & =2 \mu^{3 / 2} \int_{0}^{\sec ^{-1}(1 / \sqrt{\mu})} \sec ^{3} \vartheta d \vartheta \\
& =\mu^{3 / 2} \frac{1}{\sqrt{\mu}} \frac{\sqrt{1-\mu}}{\sqrt{\mu}}+\mu^{3 / 2} \ln \left(\frac{1}{\sqrt{\mu}}+\frac{\sqrt{1-\mu}}{\sqrt{\mu}}\right) \\
& =\sqrt{\mu} \sqrt{1-\mu}+\mu^{3 / 2} \ln \frac{\sqrt{1-\mu}+1}{\sqrt{\mu}},
\end{aligned}
$$

and $(2.12)$ is proved.

For (2.13), set $s=1+\ln (z)$. Then we have

$$
G_{2}(\nu)=\sqrt{\mu e} \int_{\mu}^{1} \frac{d z}{\sqrt{z^{2}-\mu z}}=\sqrt{\mu e} \int_{\mu}^{1} \frac{d z}{\sqrt{(z-\mu / 2)^{2}-\mu^{2} / 4}} .
$$

Set $z-\mu / 2=\mu \sec \vartheta / 2$. Then

$$
\begin{aligned}
G_{2}(\nu) & =\sqrt{\mu e} \int_{0}^{\sec ^{-1}(2 / \mu-1)} \sec \vartheta d \vartheta \\
& =\sqrt{\mu e} \ln \left\{\sqrt{(2 / \mu-1)^{2}-1}+(2 / \mu-1)\right\} \\
& =2 \sqrt{\mu e} \ln \frac{\sqrt{1-\mu}+1}{\sqrt{\mu}}
\end{aligned}
$$

for $\nu=1+\ln \mu$ with $\mu \in(0,1)$.

By the definition of $K(\mu)$, we have

$$
\begin{aligned}
K(\mu) & =\frac{1}{2}\left[\sqrt{\mu(1-\mu)}+\left(\mu^{3 / 2}+4 \mu^{1 / 2}\right) \ln \frac{\sqrt{1-\mu}+1}{\sqrt{\mu}}\right]^{2}, \\
K^{\prime}(\mu) & =\left[\sqrt{\mu(1-\mu)}+\left(\mu^{3 / 2}+4 \mu^{1 / 2}\right) \ln \frac{\sqrt{1-\mu}+1}{\sqrt{\mu}}\right] Q(\mu),
\end{aligned}
$$

where

$$
Q(\mu):=-\frac{3 \mu+3}{2 \sqrt{\mu(1-\mu)}}+\frac{3 \mu+4}{2 \sqrt{\mu}} \ln \frac{\sqrt{1-\mu}+1}{\sqrt{\mu}} .
$$

Hence $K^{\prime}(\mu)=0$ if and only if $\mu$ satisfies

$$
I(\mu):=\frac{3 \mu+3}{(3 \mu+4) \sqrt{1-\mu}}=\ln \frac{\sqrt{1-\mu}+1}{\sqrt{\mu}}:=J(\mu) .
$$

Note that $I\left(0^{+}\right)=3 / 4, I\left(1^{-}\right)=+\infty, J\left(0^{+}\right)=+\infty$, and $J\left(1^{-}\right)=0$. Moreover, $I$ is strictly increasing and $J$ is strictly decreasing. It follows that equation (2.14) has exactly one solution in $(0,1)$, say at $\mu^{*}$. Since $K(\mu) \rightarrow 0$ as either $\mu \rightarrow 0$ or $\mu \rightarrow 1, K(\mu)$ should attain its maximum at $\mu^{*}$. Therefore, $\lambda=K(\mu)$ is increasing for $0<\mu<\mu^{*}$ and $\lambda=K(\mu)$ is decreasing for $\mu^{*}<\mu<1$.

Conversely, for a given $\mu \in(0,1)$, we first compute $G_{1}(\mu)$ and $G_{2}(\nu)$ by using (2.12) and (2.13). Then we can compute $Y$ and $\lambda$ by using (2.10) and (2.11). Finally, the 
solution $U(x)$ of (2.1) - (2.2) with $U(0)=\mu$ can be computed from the formula

$$
\int_{\mu}^{U(x)} \frac{d s}{\sqrt{F_{1}(s)-F_{1}(\mu)}}=\int_{\mu}^{U(x)} \frac{\sqrt{\mu s}}{\sqrt{s-\mu}} d s=\frac{\sqrt{2 \lambda}}{1+Y} x
$$

for $x \in[0,1]$ and $U(-x)=U(x)$ for $x \in[-1,0)$.

We thus have proved the following theorem.

Theorem 2.1. There exists a positive constant $\lambda^{*}$, which is given by $\lambda^{*}=K\left(\mu^{*}\right)$, such that there are no solutions of (2.1)-(2.2) if $\lambda>\lambda^{*}$; there is exactly one solution of (2.1)-(2.2) if $\lambda=\lambda^{*}$; and there are two solutions of (2.1)-(2.2) if $\lambda<\lambda^{*}$.

Note that $\mu^{*} \approx 0.395809637819479$ and $\lambda^{*} \approx 5.64837452749789$.

3. Global existence. First, we recall from [13] that the solution $v$ of the local problem

$$
\begin{aligned}
& v_{t}=v_{x x}-\sigma v^{-2},-1<x<1, t>0, \\
& v( \pm 1, t)=1, t>0, \\
& v(x, 0)=v_{0},-1 \leq x \leq 1,
\end{aligned}
$$

exists globally in time for any positive initial data $v_{0}$ which is greater than the corresponding larger stationary solution, denoted by $w_{\sigma}^{+}$, of (2.3), if $\sigma \in\left(0, \sigma^{*}\right)$. Moreover, we have $v(x, t) \rightarrow w_{\sigma}^{+}(x)$ as $t \rightarrow \infty$ uniformly for $x \in[-1,1]$. We remark that the proof of the above convergence of $v$ given in [13] follows from an important fact that $v_{t}<0$. In particular, by differentiating equation (3.1) with respect to $t$, we obtain that

$$
\left(v_{t}\right)_{t}-\left(v_{t}\right)_{x x}-2 \sigma v^{-3} v_{t}=0 .
$$

Since $v_{t}(x, 0)=-\sigma<0$ for $x \in(-1,1)$ for the case when $v_{0} \equiv 1$, it follows from the maximum principle that $v_{t}<0$ in $(-1,1) \times(0, \infty)$. Unfortunately, we are unable to prove this monotonicity in time for our nonlocal case. Nevertheless, we have

Theorem 3.1. The solution $u$ of (1.1) with $u_{0} \equiv 1$ exists globally (in time) for any $\lambda \in\left(0,9 \sigma^{*}\right)$.

Proof. Note that $g(t ; u) \in(0,1 / 9)$ for any $t>0$, since $u<1$ for $x \in(-1,1)$. Let $v$ be the solution of (3.1)-(3.3) with $\sigma=\lambda / 9$ and $v_{0} \equiv 1$. Then from

$$
(u-v)_{t}-(u-v)_{x x}=\sigma v^{-2}-\lambda g u^{-2} \geq \sigma\left(v^{-2}-u^{-2}\right)=\sigma(u+v)(u-v) /(u v)^{2}
$$

and by applying the maximum principle, we deduce that $u \geq v$. It follows that $u$ exists globally in time, if $\lambda \in\left(0,9 \sigma^{*}\right)$.

To study the asymptotic behavior of the global solution $u$ of (1.1), we introduce the following energy functional:

$$
E(t):=\frac{1}{2} \int_{-1}^{1} u_{x}^{2}(x, t) d x+\lambda\left(1+\int_{-1}^{1} u^{-1}(x, t) d x\right)^{-1} .
$$

Then we compute that

$$
E^{\prime}(t)=-\int_{-1}^{1} u_{t}^{2}(x, t) d x
$$


by using the fact $u_{t}( \pm 1, t)=0$ and an integration by parts. Hence we have

$$
0 \leq \int_{0}^{T} \int_{-1}^{1} u_{t}^{2}(x, t) d x d t=E(0)-E(T) \leq E(0)<\infty
$$

for any $T>0$.

Note that $u$ is symmetric with respect to $x=0$ and $u_{x}<0$ in $(-1,0) \times(0, \infty)$. Again, let $v$ be the solution of (3.1)-(3.2) with $\sigma=\lambda / 9$ and $v_{0} \equiv 1$. Since $v_{t}<0$ and $v(x, t) \rightarrow w_{\sigma}^{+}(x)$ as $t \rightarrow \infty$ uniformly for $x \in[-1,1]$, we have $v(0, t) \geq w_{\sigma}^{+}(0)>0$ for any $t>0$. This implies that $u$ has a positive lower bound $w_{\sigma}^{+}(0)$ for $(x, t) \in[-1,1] \times[0, \infty)$. Then, by the parabolic regularity theory, we have the uniform boundedness for $u_{x}, u_{x x}$, and $u_{t}$ in $[-1,1] \times[0, \infty)$.

Now, taking any sequence $\left\{t_{n}\right\}$ with $t_{n} \rightarrow \infty$ as $n \rightarrow \infty$ and setting $u_{n}(x, t):=$ $u\left(x, t+t_{n}\right)$, using the above regularity result as well as the energy functional defined above, it is easy to show that $u(x, t)$ converges to one of the steady states for the given $\lambda$ (cf. e.g., [7, 8]). Note that $u(0, t) \geq v(0, t) \geq w_{\sigma}^{+}(0) \geq w_{\sigma^{*}}(0)=U_{\lambda^{*}}(0)>U_{\lambda}^{-}(0)$ for all $\lambda<\lambda^{*}$. Hence the limit must be the larger steady state, denoted by $U_{\lambda}^{+}$, of (2.1)-(2.2). Since the limit is independent of the sequence $\left\{t_{n}\right\}$, it follows that $u(x, t) \rightarrow U_{\lambda}^{+}(x)$ as $t \rightarrow \infty$.

Therefore, we have proved the following theorem.

Theorem 3.2. For any $\lambda \in\left(0,9 \sigma^{*}\right)$, the solution $u$ of (1.1) with $u_{0} \equiv 1$ converges to the steady state $U_{\lambda}^{+}$as $t \rightarrow \infty$.

4. Quenching. In this section, we shall determine whether solutions of (1.1) quench in finite time. It is well-known that, under certain conditions on the initial data, the profile of the local problem (3.1)-(3.3) at the quenching time $T$ at the quenching point $x=0$ satisfies

$$
v(x, T) \sim C\left(\frac{|x|}{\sqrt{|\ln | x||}}\right)^{2 / 3} \quad \text { as }|x| \rightarrow 0
$$

for some positive constant $C$ (see [5]). In the following theorem, we also prove that there is a cusp at the quenching point $x=0$. In the proof, the auxiliary function we used is very simple. But, we have to adapt this method to nonlocal integral terms, which we believe is quite original.

Theorem 4.1. Suppose that $u_{0} \in C^{2}[-1,1]$, and, for some $c_{0}>0$,

$$
u_{0}(x)=u_{0}(-x), \quad u_{0}(x) \geq c_{0}, \quad u_{0}^{\prime \prime}(x) \geq c_{0}, \quad u_{0}(-1)=u_{0}(1)=1 .
$$

If $\lambda$ is sufficiently large, then $u(x, t)$ quenches at $x=0$ at a finite time $T \leq 1$. Furthermore, there is a cusp at the quenching point $x=0$ : for any $2<\beta<3$, there exists $\delta_{0}=\delta_{0}(\beta)>0$ such that

$$
u(x, t) \geq \delta_{0}|x|^{2 / \beta} \quad \text { for } \quad(x, t) \in[-1,1] \times[0, T) .
$$

Proof. Let $T$ be the maximal existence time of $u$ such that $u>0$ for $t \in[0, T)$. For contradiction, we assume that $T>1$. We set $T=2$ if $T \geq 2$. 
By comparison principle, it is clear that

$$
u(x, t) \leq \varphi(x, t) \quad \text { for }-1<x<1, \quad 0<t<T,
$$

where $\varphi$ is the solution of

$$
\begin{aligned}
& \varphi_{t}=\varphi_{x x}, \quad-1<x<1,0<t<2, \\
& \varphi(-1, t)=\varphi(1, t)=1, \quad 0<t<2, \\
& \varphi(x, 0)=u_{0}(x), \quad-1<x<1 .
\end{aligned}
$$

It follows that

$$
u_{x}(1, t) \geq \varphi_{x}(1, t) \geq \varepsilon_{0}>0 \text { for } 0<t<T,
$$

where the constant $\varepsilon_{0}$ is clearly independent of $\lambda$. We choose $\varepsilon_{0}$ small enough so that

$$
g(0 ; u)>\sigma_{0}:=\left[1+\frac{2 \beta}{\beta-1}\left(\frac{2}{\varepsilon_{0}}\right)^{1 / \beta}\right]^{-2}
$$

and both (4.5) and the condition

$$
\varepsilon_{0} \leq \beta c_{0}^{\beta}
$$

hold.

Take $2<\beta<3$, fixed. It is clear that $v=u^{\beta}$ satisfies

$$
\left(v_{x}\right)_{t}-\left(v_{x}\right)_{x x}=v^{-1} v_{x} \cdot\left[(3-\beta) \lambda g(t ; u) v^{1-3 / \beta}-\frac{2(\beta-1)}{\beta}\left(v_{x}\right)_{x}\right]+\frac{\beta-1}{\beta} \frac{v_{x}^{3}}{v^{2}}
$$

for $0<x<1,0<t<T$. Recalling that $1-3 / \beta<0, u \leq 1$ and $u_{x} \geq 0$ for $0<x<1$, we obtain

$$
\left(v_{x}\right)_{t}-\left(v_{x}\right)_{x x} \geq v^{-1} v_{x} \cdot\left[(3-\beta) \lambda g(t ; u)-\frac{2(\beta-1)}{\beta}\left(v_{x}\right)_{x}\right]
$$

for $0<x<1,0<t<T$.

We take $\lambda$ large enough so that $\lambda \sigma_{0} \geq 3$ and

$$
(3-\beta) \lambda \sigma_{0}-\frac{2(\beta-1)}{\beta} \varepsilon_{0}>0 .
$$

Let $[0, \tilde{T}) \subset[0, T)$ be the maximal interval on which

$$
(3-\beta) \lambda g(t ; u)-\frac{2(\beta-1)}{\beta} \varepsilon_{0}>0, \quad 0<t<\tilde{T} .
$$

Notice that, using (4.8), we have

$$
w_{t}-w_{x x}+\frac{2(\beta-1)}{\beta} \frac{v_{x}}{v} w_{x} \geq 0,
$$

where $w:=v_{x}-\varepsilon_{0} x$. Also, from (4.1) it follows that $u_{0}^{\prime}(x) \geq c_{0} x$, and so $v_{x}(x, 0) \geq \varepsilon_{0} x$ on $[0,1]$ by (4.6). Then, using comparison on the region $(0,1) \times(0, \tilde{T})$, we find that

$$
v_{x}(x, t) \geq \varepsilon_{0} x \quad \text { for } \quad 0<x<1, \quad 0<t<\tilde{T} .
$$


It follows that

$$
\begin{aligned}
v(x, t) & \geq v(0, t)+\frac{1}{2} \varepsilon_{0} x^{2} \\
& >\frac{1}{2} \varepsilon_{0} x^{2} \quad \text { for } 0<x<1, \quad 0<t<\tilde{T},
\end{aligned}
$$

and hence

$$
u(x, t)>\delta_{0} x^{2 / \beta} \quad \text { for } 0<x<1, \quad 0<t<\tilde{T},
$$

where $\delta_{0}=\left(\varepsilon_{0} / 2\right)^{1 / \beta}$ is clearly independent of $\lambda$. This inequality implies that

$$
\int_{-1}^{1} u^{-1}(x, t) d x=2 \int_{0}^{1} u^{-1}(x, t) d x<2 \delta_{0}^{-1} \int_{0}^{1} x^{-2 / \beta} d x=\frac{2 \beta}{\beta-2} \delta_{0}^{-1},
$$

and hence

$$
g(t ; u)>\sigma_{0} \text { for } 0<t<\tilde{T} .
$$

Using (4.7) and the maximality of $[0, \tilde{T})$ we find that $\tilde{T}=T$.

Having established this lower bound for $g$, we can now apply the comparison principle to conclude

$$
u(x, t) \leq \psi(x, t) \quad \text { for } \quad-1<x<1, \quad 0<t<T,
$$

where $\psi$ is the solution of

$$
\begin{aligned}
& \psi_{t}=\psi_{x x}-\lambda \sigma_{0}, \quad-1<x<1,0<t<T, \\
& \psi(-1, t)=\psi(1, t)=1, \quad 0<t<T, \\
& \psi(x, 0)=1, \quad-1<x<1 .
\end{aligned}
$$

By comparison we have

$$
\psi(x, t) \leq 1-\frac{\lambda \sigma_{0}}{3} t\left(1-x^{2}\right) \quad \text { for } \quad-1<x<1, \quad 0<t \leq 1
$$

Thus $\psi(0, t)$ must vanish before $t=3 /\left(\lambda \sigma_{0}\right)$. This implies that $T \leq 3 /\left(\lambda \sigma_{0}\right) \leq 1$, a contradiction. The proof is complete. It is clear that (4.10) is now valid for $0<t<T$.

Acknowledgement. The authors would like to thank the anonymous referee for some helpful comments.

\section{REFERENCES}

[1] G.-J. Chen and J.-S. Guo, Critical length for a quenching problem with nonlocal singularity, Methods and Applications of Analysis 5 (1998), 185-194. MR.1636562 (99i:35073)

[2] K. Deng, Dynamical behavior of solutions of a semilinear heat equation with nonlocal singularity, SIAM J. Math. Anal. 26 (1995), 98-111. MR.1311883 (95j:35105)

[3] K. Deng, M.K. Kwong, and H.A. Levine, The influence of nonlocal nonlinearities on the long time behavior of solutions of Burgers' equation, Quart. Appl. Math. 50 (1992), 173-200. MR1146631 (92k:35241)

[4] M. Fila and J. Hulshof, A note on the quenching rate, Proc. Amer. Math. Soc. 112 (1991), 473-477. MR.1055772 (92a:35090)

[5] S. Filippas and J.-S. Guo, Quenching profiles for one-dimensional semilinear heat equations, Quart. Appl. Math. 51 (1993), 713-729. MR1247436 (95b:35029)

[6] G. Flores, G. Mercado, J.A. Pelesko, and N. Smyth, Analysis of the dynamics and touchdown in a model of electrostatic MEMS, SIAM J. Appl. Math. 67 (2007), 434-446. MR.2285871 (2007k:35330)

[7] Y. Giga and R.V. Kohn, Asymptotically self-similar blow-up of semilinear heat equations, Comm. Pure Appl. Math. 38 (1985), 297-319. MR784476 (86k:35065) 
[8] J.-S. Guo, On the quenching behavior of the solution of a semilinear parabolic equation, J. Math. Anal. Appl. 151 (1990), 58-79. MR.1069448 (91g:35021)

[9] J.-S. Guo, On the quenching rate estimate, Quart. Appl. Math. 49 (1991), 747-752. MR1134750 (92j:35097)

[10] J.-S. Guo, Quenching behavior for the solution of a nonlocal semilinear heat equation, Differential and Integral Equations 13 (2000), 1139-1148. MR.1775250 (2001f:35199)

[11] J.-S. Guo and T.-M. Hwang, On the steady states of a nonlocal semilinear heat equation, Dynamics of Continuous, Discrete and Impulsive Systems, Series A: Mathematical Analysis 8 (2001), 53-68. MR 1820665 (2002c:35135)

[12] H. Kawarada, On solutions of initial boundary value problem for $u_{t}=u_{x x}+\frac{1}{(1-u)}$, RIMS Kyoto U. 10 (1975), 729-736. MR0385328 (52:6192)

[13] H.A. Levine, Quenching, nonquenching, and beyond quenching for solution of some parabolic equations, Ann. Mat. Pura Appl. 155 (1989), 243-260. MR1042837 (91m:35028)

[14] J.A. Pelesko, Mathematical modeling of electrostatic MEMS with tailored dielectric properties, SIAM J. Appl. Math. 62 (2002), 888-908. MR.1897727 (2003b:74025)

[15] J.A. Pelesko, A.A. Triolo, Nonlocal problems in MEMS device control, J. Eng. Math. 41 (2001), 345-366. MR.1872152 